\title{
Lesões no joelho de atletas: período de destreinamento*
}

\author{
[Artículos]
}

\author{
Lídia Gontijo Vieira ${ }^{* *}$ \\ Maria Eliza Martins Ferreira ${ }^{* * *}$ \\ Anderson M. Cruz ${ }^{* * * *}$
}

Recebido: 23 de agosto de 2019

Aceito: 5 de outubro de 2020

Para citar:

Gontijo, L., Martins, M., Pereira, P. e Cruz, A. (2020). Lesões articulares e ligamentares no joelho de atletas relacionadas ao período de destreinamento.

Revista de Investigación Cuerpo, Cultura y Movimiento, 11(1).

https://doi.org/10.15332/2422474X.6470

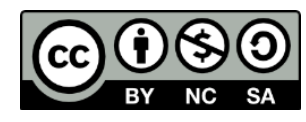

\section{Resumo}

As lesões de joelho são comuns em atletas, e a reincidência após a ocorrência da primeira lesão também acontece na maioria dos casos.

Assim, é crescente o número de estudos a esse respeito, porém há poucos

\footnotetext{
* Artigo de revisão. Não houve financimento para a pesquisa. Estudo vinculado ao Grupo de Estudos de Bioquímica, Fisiologia e Saúde da Pontifícia Universidade Católica de Goiás, Goiânia, Brasil.

** Graduanda de Educação Física na Pontifícia Universidade Católica de Goiás, Goiânia, Brasil. Correio eletrônico: lidiagontijovieira85@gmail.com; ORCID: https://orcid.org/0000-0002-7703-5514

*** Graduanda de Educação Física na Pontifícia Universidade Católica de Goiás, Goiânia, Brasil. Correio eletrônico: eliza.martinsferreira@hotmail.com; ORCID: https://orcid.org/0000-0002-7816-2304

${ }^{* * * *}$ Professor doutor da Escola de Formação de Professores e Humanidades na Pontifífica Universidade Católica de Goiás e na Universidade Estadual de Goiás, Goiânia, Brasil. Correio eletrônico: miguelcruz@pucgoias.edu.br; ORCID:

https://orcid.org/0000-0002-8116-6904
} 
estudos que interligam esse assunto e suas consequências ao período de destreino, mesmo sabendo que esse período pode proporcionar grandes perdas. Para identificar esses fatores de risco, foi realizada uma revisão bibliográfica nas bases PubMed e da Coordenação de Aperfeiçoamento de Pessoal de Nível Superior, usando a estratégia de busca PICOT. Dos 354 artigos identificados, 12 artigos atenderam aos critérios e foram analisados na íntegra, considerando a hipótese de correlação entre as lesões de joelho e o destreino. A partir disso, conclui-se que é necessário elaborar um protocolo de destreinamento prolongado para prevenir a síndrome de retirada e promover a manutenção da saúde dos atletas, principalmente daqueles que sofreram lesões de joelho. Por fim, sugerem-se ulteriores pesquisas sobre o tema para a confirmação da correlação.

Palavras-chave: atletas, saúde, destreinamento, lesões de joelho.

\section{Lesiones de rodilla en deportistas: período de desentrenamiento}

\section{Resumen}

Las lesiones de rodilla son comunes en los atletas y la recurrencia después de la primera lesión ocurre también en la mayoría de casos. Por tanto, el número de estudios sobre este tema va en aumento, pero son pocos los estudios que relacionen el tema y sus consecuencias con el período de desentrenamiento, aunque este período puede traer grandes pérdidas. Para identificar estos factores de riesgo se realizó una revisión bibliográfica en las bases de datos PubMed y la Coordenación de Perfeccionamiento de Personal de Nivel Superior, utilizando la estrategia de búsqueda PICOT. De los 354 artículos identificados, 12 cumplieron los criterios y fueron analizados en su totalidad, considerando la hipótesis de una correlación entre lesiones de rodilla y desentrenamiento. De ahí, se concluyó que es necesario elaborar un protocolo de desentrenamiento prolongado para prevenir el síndrome de abstinencia y promover el 
mantenimiento de la salud de los deportistas, especialmente los que han sufrido lesiones de rodilla. Finalmente, se sugiere una mayor investigación sobre el tema para confirmar la correlación.

Palabras clave: desportista, salud, desentrenamiento y lesiones de rodilla.

\section{Knee injuries in athletes: detraining period}

\section{Abstract}

Knee injuries are common in athletes and recurrence after the first injury also occurs in most cases. Therefore, the number of studies on this topic is increasing, but there are few studies that relate the subject and its consequences to the detraining period, although this period can bring great losses. In order to identify these risk factors, a bibliographic review was carried out in the databases PubMed and the Coordination for the Improvement of Higher Level Personnel, using the PICOT search strategy. Of the 354 articles identified, 12 met the criteria and were analyzed in their entirety, considering the hypothesis of a correlation between knee injuries and detraining. From this, it was concluded that it is necessary to develop a protocol for extended detraining to prevent withdrawal syndrome and promote the health maintenance in athletes, especially those who have suffered knee injuries. Finally, further research on the subject is suggested to confirm the correlation.

Keywords: athlete, health, detraining and knee injuries.

\section{Introdução}

Sportverletz (1999), por meio de análises realizadas durante 25 anos de atendimento ambulatorial ortopédico-traumatológico esportivo, afirma que aproximadamente $33 \%$ de todas as lesões esportivas são direcionadas ao joelho, das quais a lesão do ligamento cruzado anterior (LCA) é a mais comum entre os atletas (Wright e Splinder, 2008). 
As lesões no joelho, em particular as lesões do LCA, têm uma taxa mais alta nas seguintes modalidades: futebol, futebol de salão, futebol australiano, esqui, alpino, handebol, luta livre, rugby e basquete. Cabe salientar ainda que a proporção de lesões no LCA é maior em mulheres atletas, especialmente no basquete, no futebol e no handebol (Prodromos et al., 2007).

Griffin et al. (2000) expõem que a recorrência de lesões no LCA em mulheres relaciona-se com o tamanho do ligamento, ao alinhamento dos membros, à força muscular, aos padrões de ativação e possivelmente ao tamanho do entalhe intercondilar. O resultado da pesquisa apresenta os seguintes dados: 30\% das lesões são ocasionadas por impacto direto no joelho e 70\% são caracterizadas por lesões sem contato.

Naraghi e White (2016) identificaram um padrão de lesões ligamentares ocorridas em atletas, podendo ser elas lágrimas meniscais, lesões de ligamento cruzado posterior (LCP), lesões póstero-laterais de canto (PLC), lesões do ligamento colateral medial (LCM) e do canto póstero-medial (CPM).

As lágrimas meniscais ${ }^{1}$, quando observadas nos atletas, encontram-se de maneira isolada ou em conjunto com lesões ligamentares (Naraghi e White, 2016). Com isso, as rupturas do LCA se relacionam com as rupturas da raiz posterior dos meniscos (SMET et al., 2009). Já as lesões de LCP, quando encontradas, caracterizam-se como lesões multiligamentares e/ou isoladas ocasionadas por hiperflexão (Harner e Hoher, 1998), sendo esta uma restrição secundária à rotação externa da tíbia.

${ }^{1}$ Lágrimas meniscais são uma extrusão meniscal, edema na medula óssea subcondral adjacente, perda da cartilagem adjacente, edema perimeniscal e formação de cisto (Morrison e Major 2015).

Revista de Investigación Cuerpo, Cultura y Movimiento

ISSN: 2248-4418 | e-ISSN: 2422-474X | DOI: https://doi.org/10.15332/2422474X

Vol. 11 N. 01 | enero-junio de 2021 
Além disso, o PLC se assemelha com o LCP. São lesões ocasionadas por rotação externa em hiperextensão ou flexão, resultando em rupturas no joelho (Naraghi e White, 2016). Dessa forma, as lesões que remetem ao PLC passam muito despercebidas, sendo elas muitas vezes interligadas a outras lesões multiligamentares (Laprade et al., 2007).

No mais, as lesões de LCM são consideradas entre as lesões mais comuns do joelho (Bollen, 2000). As lesões definidas como CPM estendem-se ao aspecto posterior do LCM, superficial ao aspecto do LCP, sendo o CPM um dos estabilizadores estáticos dinâmicos. Conclui-se que as lesões do LCM são mais comuns em atletas no momento das atividades esportivas, quando o joelho está flexionado. (Naraghi e White, 2016).

Mujika e Padilha (2000) apresentam o destreino como o momento de interrupção do treinamento, podendo ocasionar a perda parcial e/ou completa das adaptações fisiológicas adquiridas durante a performance de treino. Pode-se descrever também como um intervalo de inatividade. Se, por um lado, os exercícios físicos estimulam benefícios morfológicos, fisiológicos e funcionais, por outro, o tempo necessário para que eles sejam mantidos após a interrupção do treinamento é ainda incerto. As perdas induzidas pela pausa no treinamento variam quantitativa e qualitativamente de acordo com o tempo de destreinamento (Michelin et al., 2008; Oliveira et al., 2009).

A investigação de Fatouros et al. (2004) evidencia que o treinamento pode atenuar o estresse oxidativo induzido pelo exercício em indivíduos mais velhos, aumentando a defesa antioxidante. Além disso, observou-se a redução no conteúdo de gordura corporal, o aumento da capacidade de defesa contra espécies reativas de oxigênio e nitrogênio, e melhor capitação de $\mathrm{VO}_{2}$, o que sugere que o exercício sistemático ofereça um mecanismo de proteção contra danos oxidativos e uma melhora no 
desempenho funcional (Baggish et al., 2008). No entanto, é provável que a interrupção do treinamento reverta todas essas adaptações induzidas pelo treinamento, bem como as respostas ao estresse oxidativo e ao status antioxidante. Esse princípio, conhecido também como "princípio da reversibilidade", é o princípio do destreinamento, em que os benefícios adquiridos com o treinamento físico (princípio da adaptação) quando são suspensos ou reduzidos são seguidos por um reajustamento, conforme a diminuição do estímulo (Oliveira et al., 2009; Coyle, 1994; Ruzicic et al., 2016).

E, como atletas frequentemente passam por fases de interrupção do processo de treinamento devido a doenças, lesões, férias pós-temporada ou aposentadorias, em decorrência delas, haverá também perdas fisiológicas e metabólicas, conforme descrito acima (Oliveira et al., 2009).

O destreinamento provoca também perdas das adaptações cardiovasculares e metabólicas. No treinamento resistido de longa duração, essa reversibilidade causa prejuízos no desempenho do atleta, o qual não consegue manter um exercício físico por um tempo prolongado e em uma maior intensidade. Essas reduções de desempenho já podem ocorrer a partir da primeira semana de destreino (Coyle, 1994; Fox et al., 1991; Evangelista e Brum, 1999).

Assim, o propósito deste artigo de revisão é analisar se a recorrência de lesões articulares e ligamentares no joelho está relacionada ao destreinamento ou se essas lesões podem causar mais complicações nesse período.

\section{Metodologia}

Trata-se de uma revisão de literatura na qual se utilizaram somente artigos, por meio da estratégia definida para a busca PICOT, em que P é 
paciente (os atletas), I é intervenção (lesões de joelho), C é comparador (destreino), $\mathrm{O}$ é desfecho (não utilizado) T é tempo (cinco anos).

As bases escolhidas para efetuar a busca do material foram PubMed e Coordenação de Aperfeiçoamento de Pessoal de Nível Superior. Foram utilizadas as seguintes palavras em inglês: retirement athletic injuries, knee injuries e destraining. Todos os artigos deveriam ter sido escritos de 2014 a 2019.

O material encontrado inicialmente foi constituído por 354 artigos. Após a leitura dos resumos, foram descartados os artigos que não focavam em lesões de joelho ou não falavam de atletas, o que levou a um total de 35 artigos. A partir da leitura na íntegra dos artigos selecionados, 23 foram descartados, restando 12 artigos na amostra final, conforme a figura 1 e a tabela 1.

Após concluir a análise dos artigos, os resultados foram descritos e, em seguida, foi elaborada a hipótese de correlação entre as lesões no joelho de atletas e o destreino. 
Figura 1. Amostra final.

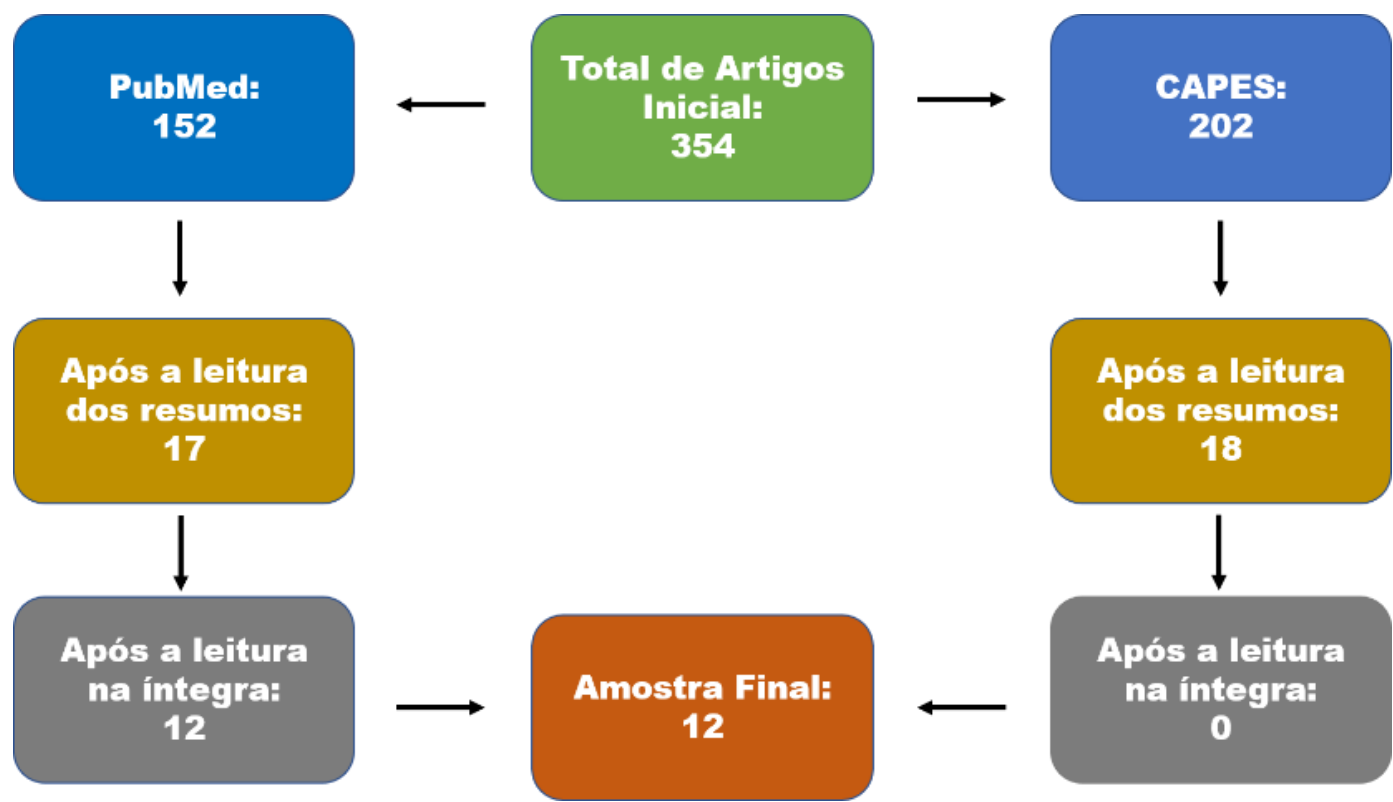

Fonte: elaboração própria.

Tabela 1. Artigos analisados

\begin{tabular}{|c|c|c|}
\hline Autor & Título & Data \\
\hline Aravindh, P. et al. & $\begin{array}{l}\text { Association of Compartmental Bone Bruise } \\
\text { Distribution with Concomitant Intra-articular and } \\
\text { Extra-articular Injuries in Acute Anterior Cruciate } \\
\text { Ligament Tears After Noncontact Sports Trauma }\end{array}$ & 2018 \\
\hline Awwad, G. E. H. et al. & $\begin{array}{l}\text { An Analysis of Knee Injuries in Rugby League: The } \\
\text { Experience at the Newcastle Knights Professional } \\
\text { Rugby League Team }\end{array}$ & 2019 \\
\hline Bonar, J. et al. & $\begin{array}{l}\text { Diferenciando o tumor de células gigantes do osso da } \\
\text { síndrome femoropatelar: um estudo de caso }\end{array}$ & 2016 \\
\hline Geblein, M. et al. & $\begin{array}{l}\text { How dangerous is American football in the German } \\
\text { amateur sector? }\end{array}$ & 2020 \\
\hline Giacomo, L. M. et al. & Management of Cyclops Syndrome: A case report & 2016 \\
\hline John, R. et al. & $\begin{array}{l}\text { Epidemiological Profile of Sports-Related Knee } \\
\text { Injuries in Northern India: An Observational Study at } \\
\text { a Tertiary Care Centre }\end{array}$ & 2016 \\
\hline $\begin{array}{l}\text { Joseph, C., Naughton, G. e } \\
\text { Antcliff. }\end{array}$ & $\begin{array}{l}\text { Australian netball injuries in 2016: An overview of } \\
\text { insurance data }\end{array}$ & 2019 \\
\hline Lutter, C. et al. & $\begin{array}{l}\text { Mecanismos de Lesões Agudas de Joelho em Atletas } \\
\text { de Bouldering e Escalada }\end{array}$ & 2020 \\
\hline
\end{tabular}

Revista de Investigación Cuerpo, Cultura y Movimiento

ISSN: 2248-4418 | e-ISSN: 2422-474X | DOI: https://doi.org/10.15332/2422474X

Vol. 11 N.o 1 | enero-junio de 2021 


\begin{tabular}{|l|l|l|}
\multicolumn{1}{|c|}{ Autor } & \multicolumn{1}{c|}{ Título } & Data \\
\hline $\begin{array}{l}\text { Morrison, W. B. e Major N. } \\
\text { Naraghi, A. e White, L. }\end{array}$ & $\begin{array}{l}\text { O joelho } \\
\text { Imagem de lesões esportivas de ligamentos do joelho e } \\
\text { meniscos }\end{array}$ & 2016 \\
\hline $\begin{array}{l}\text { Somanathan, A., Tandon, A. e } \\
\text { Yang, L. W. }\end{array}$ & $\begin{array}{l}\text { Review of magnetic resonance imaging features of } \\
\text { complications after anterior cruciate ligament } \\
\text { reconstruction }\end{array}$ & 2019 \\
\hline Vries, A. J. et al. & $\begin{array}{l}\text { The impact of patellar tendinopathy on sports and } \\
\text { work performance impactive athletes }\end{array}$ & 2017 \\
\hline
\end{tabular}

Fonte: elaboração própria.

\section{Resultados}

Bonar et al. (2016), no artigo "Diferenciando o tumor de células gigantes do osso da síndrome fêmoropatelar: um estudo de caso", apresentam um estudo de caso em que uma atleta de futebol recreativo foi diagnosticada com dor mecânica no joelho e submetida a tratamento. Ela relatava que essa condição havia começado há aproximadamente cinco anos e a atribuía ao futebol, mas, após outras avaliações e seu afastamento do esporte, descobriu-se um tumor benigno no joelho altamente agressivo. Partindo desse pressuposto, os autores destacam, no seu trabalho, como as lesões esportivas podem mascarar outras doenças. Esse caso ilustra a importância de uma reavaliação completa, levando em consideração as variáveis que fogem do bem-estar e o acompanhamento das reclamações autolimitantes persistentes. Portanto, um alto nível de suspeita em populações atléticas ou ativas deve ser exercido precocemente, a fim de evitar atrasos no diagnóstico e acelerar a recuperação.

No artigo de Aravindh et al. (2018), foram analisados 703 procedimentos de reconstrução do LCA, utilizando como critérios de inclusão a ressonância magnética, duas lesões sofridas durante atividades esportivas e três mecanismos de lesão. Após a análise, conclui-se que as lesões de joelho eram frequentes durante o futebol, o basquete, o netball, o frisbee, $o$ 
rugby, o badminton e o vôlei. O estudo levantou a hipótese de que a prevalência de lesões intra e extra-articulares estaria significativamente associada a um aumento no comprometimento da contusão óssea do compartimento lateral sozinho para os compartimentos medial e lateral do joelho. O resultado do estudo demonstrou que a distribuição compartimental de hematomas ósseos está associada a lesões intra e extraarticulares concomitantes

Para Giacomo e colaboradores (2016), a lesão do LCA é uma lesão atlética comum e uma das condições mais comumente tratadas no joelho. Esta lesão pode levar à síndrome do ciclope ${ }^{22}$, por isso os autores apresentam um relato de caso, no qual apresentam um atleta masculino de 25 anos que apresentou dor na face anterior do joelho direito e incapacidade de realizar a extensão total da articulação do joelho. Esse indivíduo tinha, no seu histórico, uma cirurgia de reconstrução do LCA feita há sete anos. O programa pós-operatório da primeira reconstrução foi padrão e, quando ele se recuperou, retornou às atividades diárias e à prática esportiva, mas, após o período citado, foi diagnosticado com síndrome do ciclope. Os autores enfatizam que essa síndrome deve ser considerada no diagnóstico diferencial em pacientes com perda de extensão do joelho, após a reconstrução do LCA.

No artigo de John et al. (2016), "Perfil epidemiológico de lesões no joelho relacionadas ao esporte no norte da Índia”, os autores desenvolveram um estudo observacional feito em um centro de atendimento terciário, com o objetivo de analisar a frequência de diferentes lesões no joelho em vários esportes no cenário indiano e avaliar os fatores que podem afetar o retorno ao esporte. Concluíram que o futebol foi o esporte em que mais atletas

\footnotetext{
2 Síndrome de ciclope é uma restrição da extensão do joelho que ocorre devido a um enxerto mal localizado, má remoção dos tecidos mortos ou até mesmo um trauma tecidual após a reconstituição cirúrgica de LCA (Giacomo et al., 2016).
}

Revista de Investigación Cuerpo, Cultura y Movimiento

ISSN: 2248-4418 | e-ISSN: 2422-474X | DOI: https://doi.org/10.15332/2422474X

Vol. 11 N. 01 | enero-junio de 2021 
apresentaram lesões de joelho, chegando a 30,6\% das lesões. A lesão mais comum foi a de LCA; em segundo lugar, foi a lesão meniscal lateral e medial.

Foi analisado também o estudo de Vries et al. (2017) sobre o impacto da tendinopatia patelar no esporte e no desempenho de outros trabalhos em atletas ativos. Os autores estudaram atletas com tendinopatia patelar que relatavam o impacto negativo substancial no desempenho esportivo, na capacidade de trabalho e na produtividade. O estudo foi feito com 77 participantes, dos quais 42 atletas foram classificados com baixo desempenho esportivo. No estudo, concluiu-se que a baixa performance estava relacionada com a lesão esportiva de tendinopatia patelar. Além disso, 28 atletas relataram que também no seu cotidiano as lesões de joelho prejudicavam muito a produtividade das suas vidas diária e em outros trabalhos.

Somanathan et al. (2019) tinham como objetivo analisar as complicações após a reconstrução do LCA, se esta realmente restaurava a função do joelho de modo que ficasse idêntica à do ligamento nativo e se impediria danos irreversíveis de longo prazo. Os autores concluíram que essas reconstruções são propensas a falhas mecânicas e estão associadas ao aumento da sinovite e osteólise. Entre quatro e oito meses após a cirurgia de reconstrução, os enxertos sofrem revascularização e apresentam sinal intermediário; após 12 meses, os enxertos passam por "ligamentização", resultando em um sinal semelhante ao LCA nativo, embora isso às vezes leve até 24 meses.

Os autores supracitados mostram que as complicações após a reconstrução do LCA podem ser categorizadas em dois grupos, um de joelho frouxo e instável, podendo haver a ruptura secundária do enxerto, e outro grupo que tem como principal sintoma a diminuição da amplitude de 
movimento, que pode ser resultado do impacto do enxerto e da artrofibrose localizada ou difusa.

O artigo de Naraghi e White (2016) é uma revisão de imagens que mostram os padrões de lesões ligamentares ocorridas em atletas, sendo que as mais comuns analisadas pelos autores foram: as lágrimas meniscais, LCP, PLC, LCM e do CPM. A partir das análises eles enfatizaram que as lesões que acontecem no ligamento cruzado posterior, por serem incomuns e isoladas, acabam sendo menosprezadas. Dessa maneira, há um retorno antecipado do atleta para o jogo, o que pode causar recorrências e problemas futuros.

O artigo de revisão de Morrison e Major (2015), intitulado de "O joelho", apresenta alguns tipos de lesões de joelho originadas por modalidade desportiva. Os autores expõem que a lesão de LCA é a mais comum entre os atletas. Alertam também que há grande variedade de patologias no joelho e que novas condições continuam a ser descritas, enquanto as condições das patologias estabelecidas também evoluem. Por isso, a grande necessidade de mais estudos para a adequação de diagnósticos.

Lutter et al. (2020) apresentam os mecanismos de lesões agudas de joelho em atletas de bouldering e escaladas, por meio de um estudo de caso que analisa as lesões pertinentes em atletas escaladores competitivos e não competitivos. Os pacientes tinham lesões agudas no joelho referente a escaladas ou pedregulho e de quedas no chão. O principal dano estrutural no joelho foi uma lesão meniscal medial, que foi o principal diagnóstico em $28,6 \%$ de todas as lesões, essas lesões foram causadas na maioria das vezes pelas posições de degrau alto, queda de joelho e gancho no calcanhar. Lesões do LCA combinadas com ligamento colateral medial e lesões meniscais mediais foram detectados em 9,1\% e lágrimas isoladas do LCA ou lágrimas parciais do LCA, em 2,6\%. 
No estudo de Geblein et al. (2020), verificou-se que a maioria das lesões ocorreu no treinamento e $27,3 \%$ de todas as lesões se referiam a lesões no joelho. O futebol americano é um esporte altamente propicio a lesões, e as articulações do joelho são sempre as mais afetadas, por isso foi recomendado aos atletas que tivessem um acompanhamento médico contínuo e estreito, com intuito de reduzir essas taxas de lesões.

O artigo de Joseph et al. (2019) sobre lesões em atletas australianos de netball mostra que 42\% (509 de 1000 casos analisados) da amostra apresenta atletas com lesões no joelho.

Por fim, Awwad et al. (2019), na análise das lesões de joelho na liga de rugby, tiveram o intuito de analisar os mecanismos dessas lesões, as intervenções necessárias e o tempo de recuperação, comparando-os com dados semelhantes da literatura. Foi observado que a maioria dos jogadores no estudo sofreu uma lesão no joelho, com uma incidência de 616,7 lesões por 1000 jogadores, sendo que o tempo médio levado para o retorno aos treinos de todas as lesões foi de três semanas, mas as lesões do LCA foram responsáveis por um retorno mais demorado. Um dos jogadores que sofreu uma lesão no LCA voltou a jogar na liga de rugby da primeira série, após sua ruptura inicial, mas ainda estava em reabilitação e, por isso, houve uma ruptura do enxerto. As lágrimas meniscais também fizeram com que os jogadores perdessem mais tempo para retornar aos jogos (mediana de 236 dias).

Os mesmos autores observaram que houve 73 novas lesões durante $o$ tempo do estudo e 16 foram recorrentes e um dos jogadores sofreu uma ruptura do enxerto do LCA após dois anos da reconstrução inicial. O resultado do estudo foi que a maioria das lesões foram adquiridas durante o treinamento e algumas lesões se assemelham com lesões que ocorrem comumente em outras modalidades. 
De acordo com os resultados apresentados, nota-se que lesões de joelho são comuns em atletas de variadas modalidades e há grande reincidência após a ocorrência da primeira lesão. No entanto, a literatura que correlaciona essas lesões com os períodos de destreino são escassas, o que dificultou e limitou a presente pesquisa; por isso, ao fazer a análise dos resultados encontrados, foi levantada a hipótese de correlação entre lesões no joelho de atletas e destreino.

Awwad et al. (2019) e Aravindh et al. (2018) afirmam que as recorrências de lesões são constantes, por exemplo, o caso do jogador que sofreu uma lesão no LCA e voltou a jogar na liga de rugby, mesmo ainda estando em reabilitação, e subsequentemente sofreu uma ruptura do enxerto. Há a hipótese de que as perdas ocasionadas pelo período de destreinamento após a primeira cirurgia de reconstituição do LCA possam corroborar para que o atleta seja acometido por essas recorrências, caso não haja um retorno pensado e programado à atividade de treinamento, pois esse período de destreinamento leva à perda parcial ou completa dos ajustamentos anatômicos, fisiológicos e das adaptações cardiovasculares e metabólicas adquiridos no treinamento físico (Michelin et al., 2008; Oliveira et al., 2009; Pedlar et al., 2018; Pelliccia et al., 2002; Maron, 1993; Chi et al., 1983).

Vries et al. (2017) relatam que essas lesões e recorrências podem causar dificuldades na vida cotidiana desses atletas, o que é confirmado no estudo apresentado por Oliveira et al. (2009) em que o objetivo era avaliar os efeitos de 40 dias de destreinamento sobre variáveis de força máxima, força explosiva e velocidade, em jovens atletas de handebol do sexo feminino.

As atletas foram avaliadas $48 \mathrm{~h}$ a partir do último jogo do ano e reavaliadas após 40 dias (período de destreinamento). Os resultados entre o período 
de treinamento e o período de destreinamento no teste de corrida de 20m, foi um aumento significativo no tempo necessário para completar os 20m, além disso houve uma grande redução na força máxima do agachamento. Essas perdas de força máxima e adaptações cardiovasculares, e as consequências prejudiciais causadas por lesões no joelho podem aumentar o desconforto apresentado por Vries et al. (2017).

Conforme também foi relatado no estudo de Somanathan et al. (2019) como consequência da reconstrução do LCA, o atleta pode ficar com o joelho frouxo e instável, podendo causar a ruptura do enxerto feito na reconstrução. Pode haver também uma diminuição da amplitude de movimento, que pode ser resultado do impacto do enxerto e da artrofibrose localizada ou difusa. Essas lesões atléticas são muito comuns, sendo uma das condições mais tratadas do joelho, e podem levar à síndrome do ciclope, mesmo após anos da reconstrução (Giacomo et al., 2016; Morrison e Major, 2015 ; Balcarek et al., 2008; Noailles et al., 2019; Delaloye et al., 2020). Essas consequências podem causar também um tipo de osteoartrite articular, que ocorre quando o tecido flexível nas extremidades dos ossos do joelho se desgasta, isso é particularmente importante, pois lesões anteriores no joelho são um precursor bemreconhecido da osteoartrite articular e existe uma forte possibilidade de que uma incidência crescente de lesões esportivasresulte em uma carga futura cada vez maior de osteoartrite articular, após a aposentadoria desses atletas (Finch et al., 2015; Bennell et al., 2012; Lohmander et al., 2007).

Em atletas, há também um risco aumento de osteoartrite articular, segundo os autores supracitados, devido ao estresse biomecânico repetido e aos osteófitos apresentados nas radiografias, que muitas vezes não são considerados um achado deletério, mas representam um esforço que o 
atleta faz para estabilizar as articulações. Por isso, atletas que se submetem a cirurgias de joelho e que ainda não se recuperaram das adaptações fisiomorfológicas do período de destreino devem retornar desse período de forma passiva e reabilitadora, pois, caso não ocorra correta cicatrização das articulações, ligamentos e tendões, haverá um risco aumentado de que no futuro se desenvolva osteoartrite articular (Saxon et al., 1999).

Essas comorbidades causadas por lesões esportivas podem levar também a um destreino forçado, que inclui absenteísmo, perda de produtividade, aposentadoria precoce e morte prematura. Além disso, a osteoartrite articular é um preditor independente do aumento do risco de doenças cardiovasculares pelo qual as pessoas submetidas a correções articulares têm 26\% mais chances de apresentar doenças cardiovasculares do que as pessoas sem osteoartrite articular (Finch et al., 2015; Roos, 2005; Rahman, 2013).

A osteoartrite articular está associada também a uma redução nas atividades físicas, nas atividades diárias e principalmente na qualidade de vida, o que contribui diretamente para a redução da produtividade devido ao aumento das taxas de absenteísmo e presenteísmo, à função física deficiente e à fadiga secundária a distúrbios do sono (Finch et al., 2015). Estudos nos quais foram avaliados o impacto do destreinamento, originado pela fase de transição, na antropometria e no desempenho de atletas de pentatlon moderno e natação, encontraram-se diferença significativa na massa corporal total e nas dobras cutâneas, e alterações consideráveis na função muscular do tronco, no equilíbrio e no desemprenho, no período de 25 a 45 de destreino (Fonseca et al., 2014; Sedaghati et al., 2018).

Os estudos do resultado apresentados também mostraram que as lesões no joelho acontecem em grandes escalas nos esportes como futebol, futebol 
americano, handbol, ruby e vôlei, mas estão presentes em esportes menos conhecidos como no netball, no bouldering e nas escaladas (Aravindh et al., 2018; Joseph et al., 2019.; Geblein et al., 2020; Lutter et al., 2020), e que essas lesões muitas vezes são reincidentes, logo esses atletas são candidatos a terem osteoartrite articular.

Nesse sentido, deve ser feita uma transição planejada entre o período de treino e o de destreino, ocasionado pelas lesões do joelho, para que haja a recuperação completa do estado psíquico-funcional do atleta, com total ou parcial redução das cargas de treinamento. Isso levaria a um processo de destreinamento responsivo, o qual diminuiria as perdas apresentadas e preveniria novas lesões (Fonseca et al., 2014).

Alguns estudos comprovam que, quando se combinam cargas altas de treinamento resistido com treinamento de intensidade aeróbica mais baixo, há um maior ganho de força, e as perdas descritas que ocorrem após duas a quatro semanas de destreinamento é menor (Sousa et al., 2009; Joo, 2018). A redução gradual de dias de treinamento, intensidade e volume também pode ser uma estratégia para minimizar as perdas nesse período (Hickson e Rosenkoetter, 1981; Shepley et al. 1985; Gibala et al., 1994).

Há relatos ainda de condições que foram diagnosticadas erroneamente ou após longos períodos (entre cinco e sete anos), quando os atletas já estavam aposentados (destreino), já que o esporte e a recorrência de lesões mascaravam a real patologia (Bonar et al., 2016; Morrison e Major 2015). Portanto, é importante que sejam realizados mais estudos da relação entre lesões sofridas no período de grande atividade e destreinamento, pois há grande variedade de patologias no joelho. Além disso, novas condições continuam a ser apresentadas, enquanto as condições das que já estão estabelecidas evoluem (Morrison e Major, 2015). 


\section{Conclusões}

Deverá ser planejada uma forma correta de se fazer o destreinamento prolongado após o encerramento da carreira esportiva, não somente com a finalidade de evitar a síndrome da retirada mas também de manter a saúde dos atletas, principalmente dos que sofreram lesões de joelho, o que contribuiria para melhorar a qualidade de vida desses atletas na sua vida cotidiana.

Sugere-se que novos estudos sejam feitos com o tema apresentado nesta revisão a fim de buscar uma melhor compreensão das consequências das lesões sofridas por atletas e do período de destreino, para que este seja feito de forma mais responsiva.

\section{Referências}

Aravindh, P., Wu, T., Chan C. X., Wong K. L. e Krishna L. (2018). Association of compartmental bone bruise distribution with concomitant intra-articular and extra-articular injuries in acute anterior cruciate ligament tears after noncontact sports trauma. Orthopaedic Journal of Sports Medicine, 6(4), 1- 9.

https://doi.org/10.1177/2325967118767625

Awwad, G. E. H., Coleman, J. H., Dunkley C. J. e Dewar D. C. (2019). An analysis of knee injuries in Rugby League: The experience at the Newcastle Knights Professional Rugby League Team. Sports Med - Open, 5, 33-1-7.

\section{https://doi.org/10.1186/s40798-019-0206-Z}

Baggish, A. L., Wang, F., Weiner, R. B., Elinoff, J. M., Tournoux, F., Boland, A., Picard, M. H., Hutter, A. M., Jr e Wood, M. J. (2008). Training-specific changes in cardiac structure and function: A prospective and longitudinal assessment of competitive athletes. Journal of applied physiology (Bethesda, Md.: 1985), 104(4), 1121-1128. https://doi.org/10.1152/japplphysiol.01170.2007

Balcarek, P., Sawallich, T., Losch, A., Stürmer, K. M. e Frosch, K. H. (2008). Delayed cyclops syndrome: Symptomatic extension block four years after anterior cruciate ligament reconstruction. Acta orthopaedica Belgica, 74(2), 261-265. 
Bennell, K., Hunter, D. J. e Vicenzino, B. (2012). Long-term effects of sport: Preventing and managing OA in the athlete. Nature reviews. Rheumatology, 8(12), 747-752. https://doi.org/10.1038/nrrheum.2012.119

Bollen, S. (2000). Epidemiologia das Lesões no Joelho: diagnóstico e triagem. Br J Sports Med, 34(3), 227-228. http://dx.doi.org/10.1136/bjsm.34.3.227-a

Bonar, J., Carr, S. C., de Carvalho, D. e Wunder, J. S. (2016). Differentiating giant cell tumor of bone from patellofemoral syndrome: A case study. The Journal of the Canadian Chiropractic Association, 6o(1), 57-65. https://europepmc.org/article/med/27069267

Chi, M. M., Hintz, C. S., Coyle, E. F., Martin, W. H., Ivy, J. L., Nemeth, P. M., Holloszy, J. O. e Lowry, O. H. (1983). Effects of detraining on enzymes of energy metabolism in individual human muscle fibers. The American journal of physiology, 244(3), C276-C287. https://doi.org/10.1152/ajpcell.1983.244.3.C276

Coyle, E. F. (1994). Destreinamento e retenção das adaptações induzidas pelo treinamento. Em American College of Sport Medicine. Prova de esforço e prescrição de exercício (pp. 80-86). Revinter.

Delaloye, J. R., Murar, J., Vieira, T. D., Franck, F., Pioger, C., Helfer, L., Saithna, A. e Sonnery-Cottet, B. (2020). Knee extension deficit in the early postoperative period predisposes to cyclops syndrome after anterior cruciate ligament reconstruction: A risk factor analysis in 3633 patients from the SANTI study group database. The American journal of sports medicine, 48(3), 565-572. https://doi.org/10.1177/0363546519897064

Evangelista, F. S. A. e Brum, P. C. (1999). Efeitos do destreinamento físico sobre a "performance" do atleta: uma revisão das alterações cardiovasculares e músculoesqueléticas. Revista Paulista de Educação Física, 13(2), 239-249. https://doi.org/10.11606/issn.2594-5904.rpef.1999.137873

Fatouros, I. G., Jamurtas, A. Z., Villiotou, V., Pouliopoulou, S., Fotinakis, P., Taxildaris, K. e Deliconstantinos, G. (2004). Oxidative stress responses in older men during endurance training and detraining. Medicine and science in sports and exercise, 36(12). https://doi.org/10.1249/01.mss.0000147632.17450.ff 
Finch, C. F., Kemp, J. L. e Clapperton, A. J. (2015). The incidence and burden of hospitaltreated sports-related injury in people aged 15+ years in Victoria, Australia, 20042010: A future epidemic of osteoarthritis? Osteoarthritis and cartilage, 23(7), 1138-1143. DOI https://doi.org/10.1016/j.joca.2015.02.165

Fonseca, J., Loureiro, L. L., Feital E. M. e Pierucci A. P. T. (2014). Impacto do destreinamento na antropometria de adolescentes pentatletas. Rev Bras Med Esporte, 20, 398-34. https://doi.org/10.1590/1517-86922014200501903

Fox, E. L., Bowers, R. W. e Foss, M. L. (1991). Bases fisiológicas da educação física e dos desportos ( $4^{\mathrm{a}}$ ed.). Editora Guanabara Koogan.

http://www.bibliotecadigital.unicamp.br/document/?view=000317292

Geblein, M., Frodl A., Millrose M., Biber R., Bail H. J. e Wolpert A. K. (2020). How dangerous is American football in the German amateur sector? An Analysis of positional injury over four seasons. Unfallchirurg, 123, 473-478.

https://doi.org/10.1007/s00113-019-00743-Z

Giacomo, L. M., Khan M. S., Piscitelli L., Bisaccia M. e Caraffa A. (2016). Management of cyclops syndrome: A case report. $J$ Pak Med Assoc, 66, 1330-1333. https://jpma.org.pk/article-details/7943?article id=7943

Gibala, M. J., Macdougall, J. D. e Sale, D. G. (1994). The effects of tapering on strength performance in trained athletes. International journal of sports medicine, 15(8), 492-497. https://doi.org/10.1055/s-2007-1021093

Griffin, L. Y., Agel, J., Albohm, M. J., Arendt, E. A., Dick, R. W., Garrett, W. E., Garrick, J. G., Hewett, T. E., Huston, L. M. S., Irlanda, M. L., Johnson, R. J., Kibler, W. B., Lephart, S., Lewis, J. L., Lindenfeld, T. N., Mandelbaum, B., Marchak, P., Teitz, C. C. e Wojtys, E. M. (2000). Lesões sem contato do ligamento cruzado anterior: fatores de risco e estratégias de prevenção [Noncontact Anterior Cruciate Ligament Injuries: Risk Factors and Prevention Strategies]. Journal of the American Academy of Orthopaedic Surgeons, 8, 141-150.

https://journals.lww.com/jaaos/fulltext/2000/05000/noncontact anterior cruci ate ligament injuries .1.aspx

Harner, C. D. e Höher J. (1998). Avaliação e tratamento de lesões do ligamento cruzado posterior. Am J Sports Med, 26, 471-482.

https://doi.org/10.1177\%2F03635465980260032301

Revista de Investigación Cuerpo, Cultura y Movimiento

ISSN: 2248-4418 | e-ISSN: 2422-474X | DOI: https://doi.org/10.15332/2422474X

Vol. 11 N.o 1 | enero-junio de 2021 
Hickson, R. C. e Rosenkoetter, M. A. (1981). Reduced training frequencies and maintenance of increased aerobic power. Medicine and science in sports and exercise, 13(1), 13-16.

John, R., Dhillon, M. S, Syam, K., Prabhakar, S., Behera P. e Singhb, H. (2016). Epidemiological profile of sports-related knee injuries in northern India: An observational study at a tertiary care centre. Clin Orthop Trauma, 7, 207-211. https://doi.org/10.1016/j.jcot.2016.02.003

Joo C. H. (2018). The effects of short term detraining and retraining on physical fitness in elite soccer players. PloS one, 13(5), e0196212. https://doi.org/10.1371/journal.pone.0196212

Joseph, C., Naughton, G. e Antcliff, A. (2019). Australian netball injuries in 2016: An overview of insurance data. Journal of science and medicine in sport, 22(12), 13041308. https://doi.org/10.1016/j.jsams.2019.07.016

Laprade R. F., Wentorf F. A., Fritts H., Gundry C. e Hightower C. D. (2007). Um estudo prospectivo de ressonância magnética da incidência de lesões posterolaterais e de ligamentos múltiplos em lesões agudas do joelho com hemartrose. Arthroscopy, 23(12),1341-1347. https://doi.org/10.1016/j.arthro.2007.07.024

Lohmander, L. S., Englund, P. M., Dahl, L. L. e Roos, E. M. (2007). The long-term consequence of anterior cruciate ligament and meniscus injuries: Osteoarthritis. The American journal of sports medicine, 35(10), 1756-1769. https://doi.org/10.1177/0363546507307396

Lutter, C., Tischer, T., Cooper, C., Frank, L., Hotfiel, T., Lenz, R. e Schöffl, V. (2020). Mechanisms of acute knee injuries in bouldering and rock climbing athletes. The American journal of sports medicine, 48(3), 730-738. https://doi.org/10.1177/0363546519899931

Maron, B. J., Pelliccia, A., Spataro, A. e Granata, M. (1993). Reduction in left ventricular wall thickness after deconditioning in highly trained olympic athletes. British heart journal, 69(2), 125-128. https://doi.org/10.1136/hrt.69.2.125

Marques, M. C., Casimiro, F. L. M., Marinho, D. A. e Costa, A. F. M. M. C. D. (2011). Efeitos do treino e do destreino sobre indicadores de força em jovens voleibolistas: implicações da distribuição do volume. Motriz: Revista de Educação Física, 17(2), 235-243. https://doi.org/10.5016/1980-6574.2011v17n2p235

Revista de Investigación Cuerpo, Cultura y Movimiento

ISSN: 2248-4418 | e-ISSN: 2422-474X | DOI: https://doi.org/10.15332/2422474X

Vol. 11 N. ${ }^{\circ} 1$ | enero-junio de 2021 
Michelin, E., Coelho, C. F. e Burini, R. C. (2008). Efeito de um mês de destreinamento sobre a aptidão física relacionada à saúde em programa de mudança de estilo de vida. Revista Brasileira de Medicina do Esporte, 14(3), 192-196.

https://doi.org/10.1590/S1517-86922008000300006

Morrison, W. B. e Major, N. (2015). The knee. Topics in Magnetic Resonance Imaging, 24(4), 193-203. https://doi.org/10.1097/rmr.0000000000000059

Mujika, I. e Padilla, S. (2000). Detraining: Loss of training-induced physiological and performance adaptations. Part I: short term insufficient training stimulus. Sports medicine, 3o(2), 79-87. https://doi.org/10.2165/00007256-200030020-00002

Naraghi, A. e White L. (2016) Imaging of athletic injuries of knee ligaments and menisci. Sports Imaging Series, 281(1), 23-40. https://doi.org/10.1148/radiol.2016152320

Noailles, T., Chalopin, A., Boissard, M., Lopes, R., Bouguennec, N. e Hardy, A. (2019). Incidence and risk factors for cyclops syndrome after anterior cruciate ligament reconstruction: A systematic literature review. Orthopaedics \& traumatology, surgery \& research: OTSR, 105(7), 1401-1405.

https://doi.org/10.1016/j.otsr.2019.07.007

Oliveira, V. L., Leite, G. S., Leite, R. D., Assumpção, O. C., Pereira, G. B., Neto, J. B. e Prestes, J. (2009). Efeito de um período de destreinamento sobre variáveis neuromusculares em atletas de Handebol. Fitness \& Performance Journal, 8(2), 96-102. https://dialnet.unirioja.es/servlet/articulo?codigo $=2958724$

Pedlar, C. R., Brown, M. G., Shave, R. E., Otto, J. M., Drane, A., Michaud-Finch, J., Contursi, M., Wasfy, M. M., Hutter, A., Picard, M. H., Lewis, G. D. e Baggish, A. L. (2018). Cardiovascular response to prescribed detraining among recreational athletes. Journal of Applied Physiology (Bethesda, Md.: 1985), 124(4), 813-820. https://doi.org/10.1152/japplphysiol.oog11.2017

Pelliccia, A., Maron, B. J., De Luca, R., Di Paolo, F. M., Spataro, A. e Culasso, F. (2002). Remodeling of Left ventricular hypertrophy in elite athletes after long-term deconditioning. Circulation, 105(8), 944-949.

https://doi.org/10.1161/hco802.104534

Revista de Investigación Cuerpo, Cultura y Movimiento

ISSN: 2248-4418 | e-ISSN: 2422-474X | DOI: https://doi.org/10.15332/2422474X

Vol. 11 N.o 1 | enero-junio de 2021 
Prodromos C. C., Han, Y., Rogowski, J., Joyce, B. e Shi, K. (2007). A meta-analysis of the incidence of anterior cruciate ligament tears as a function of gender, sport, and a knee injury-reduction regimen. Arthroscopy: The journal of arthroscopic \& related surgery, 23(12), 1320-1325.e6. https://doi.org/10.1016/j.arthro.2007.07.003

Rahman, M. M., Kopec, J. A., Anis, A. H., Cibere, J. e Goldsmith, C. H. (2013). Risk of cardiovascular disease in patients with osteoarthritis: A prospective longitudinal Study. Arthritis care \& research, 65(12), 1951-1958.

https://doi.org/10.1002/acr.22092

Roos, E. M. (2005). Joint injury causes knee osteoarthritis in young adults. Current opinion in rheumatology, 17(2), 195-200. https://doi.org/10.1097/01.bor.0000151406.64393.00

Ruzicic, R. D., Jakovljevic, V. e Djordjevic, D. (2016). Oxidative stress in training, overtraining and detraining: From experimental to applied research. Serbian Journal of Experimental and Clinical Research, 17(4), 343-348. https://doi.org/10.1515/sjecr-2016-0002

Saxon, L., Finch, C. e Bass, S. (1999). Sports participation, sports injuries and osteoarthritis: Implications for prevention. Sports medicine, 28(2), 123-135. https://doi.org/10.2165/00007256-199928020-00005

Sedaghati, P., Sarlak, P. e Saki F. (2018). Comparing the effect of short-term detraining on athletic performance of elite competitive children and adolescent wimmers. Feyz, 22(1), 67-74. http://eprints.kaums.ac.ir/3271/

Shepley, B., Macdougall, J. D., Cipriano, N., Sutton, J. R., Tarnopolsky, M. A. e Coates, G. (1992). Physiological Effects of Tapering in Highly Trained Athletes. Journal of Applied Physiology (Bethesda, Md.: 1985), 72(2), 706-711. https://doi.org/10.1152/jappl.1992.72.2.706

Smet, A. A., Blankenbaker, D. G., Kijowski R., Graf, B. K. e Shinki, K. (2009). Diagnóstico por RM das rupturas radiculares posteriores do menisco lateral usando a artroscopia como padrão de referência. AJR Am J Roentgenol, 192(2), 480-486. https://www.ajronline.org/doi/full/10.2214/AJR.08.1300 
Somanathan, A., Tandon, A. e Yang, L. W. (2019). Review of magnetic resonance imaging features of complications after anterior cruciate ligament reconstruction. Singapore medical Journal, 6o(2), 63-68. https://doi.org/10.11622/smedj.2019017

Sousa, A. C., Neiva, H. P., Izquierdo, M., Cadore, E. L., Alves, A. R. e Marinho, D. A. (2019). Concurrent training and detraining: Brief review on the effect of exercise intensities. International Journal of Sports Medicine, 4O(12), 747-755.

https://doi.org/10.1055/a-0975-9471

Spindler, K. P. e Wright, R. W. (2008). Prática clínica. Lágrima do Ligamento cruzado anterior. N Engl $J$ Med, 359, 2135-2142.

http://www.revdesportiva.pt/files/FIMS standing_positions/Anterior_Cruciate Ligament Injuries Anatomy..7.pdf

Sportverletz, S. K. (1999). Epidemiologia das lesões esportivas: análise de 25 Anos do atendimento ambulatorial ortopédico-traumatológico esportivo. Thieme, 13(2), 3852. https://www.thieme-connect.com/products/ejournals/abstract/10.1055/s2007-993313

Vries, A. J., Koolhaas, W., Zwerver J., Diercks R. L., Nieuwenhuis K., Worp H. V. D., Sandra, B. e Inge Van, D. A. S. (2017). The impact of patellar tendinopathy on sports and work performance in active athletes. Research in SportsMedicine, 25(3), 253-265. https://doi.org/10.1080/15438627.2017.1314292

Revista de Investigación Cuerpo, Cultura y Movimiento ISSN: 2248-4418 | e-ISSN: 2422-474X | DOI: https://doi.org/10.15332/2422474X Vol. 11 N.o 1 | enero-junio de 2021 